Pacific Journal of Mathematic 


\section{CARACTÉRISATION SPECTRALE DES ALGÈBRES DE BANACH COMMUTATIVES}

\section{BERNARD AUPETIT}

Let $A$ be a complex Banach algebra, using subharmonic functions we extend results of Le Page, Hirschfeld and Zelazko in showing the equivalence of the four properties: $A / \operatorname{Rad} A$ is commutative, the spectral radius is uniformly continuous on $A$, the spectral radius is subadditive on $A$, the spectral radius is submultiplicative on $A$.

1. Introduction. Dans la suite $A$ désigne une algèbra de Banach complexe munie de la norme \|\| et, pour $x \in A, \rho(x)$ est le rayon spectral, c'est-à-dire $\lim _{n \rightarrow \infty} \| x^{n}||^{1 / n}$, ou encore $\operatorname{Max}|\lambda|$ pour $\lambda \in \operatorname{Sp} x$, $\delta(x)$ est le diamètre de $\operatorname{Sp} x$, c'est-à-dire $\operatorname{Max}|\lambda-\mu|$ pour $\lambda, \mu \in \operatorname{Sp} x$.

Un des premiers articles à s'intéresser à la caractérisation des algèbres de Banach commutatives est celui de C. Le Page [6]. Il y démontre, en particulier, les résultats suivants, dans le cas où $A$ est unifère:

-Si $\rho(x)=\|x\|$ quel que soit $x \in A$ alors $A$ est commutative.

-S'il existe $\alpha>0$ tel que $\|x y\| \leqq \alpha\|y x\|$ quels que soient $x, y \in A$ alors $A$ est commutative.

- Si $\rho(x y-y x)=0$ quels que soient $x, y \in A$ alors $x y-y x \in \operatorname{Rad} A$.

Un peu plus tard, R. A. Hirschfeld et W. Żelazko [5] démontrèrent par une méthode absolument identique à celle de Le Page que, si $A$ est quelconque et si $\alpha\|x\| \leqq \rho(x)$ pour tout $x \in A$, alors $A$ est commutative et même, c'est une algèbre de fonctions, c'est-à-dire une sous-algèbre fermée de $\mathscr{C}(X)$, pour un certain $X$ localement compact, avec la norme équivalente $\rho(x)$. Dans cet article ils énoncèrent deux conjectures qui, malheureusement, ne sont pas encore résolues.

Conjecture 1. Si $\|$ \| et $\rho$ sont équivalents sur toute sous-algèbre commutative de $A$, alors $A$ est commutative.

Conjecture 2. Si $\rho$ est continu sur $A$ et $\rho(x)=0$ impliques $x=0$ alors $A$ est commutative.

Depuis, on a donné des exemples d'algèbres non commutatives où $\rho(x)=0$ implique $x=0$ (voir par exemple J. Duncan et A. W. Tullo [2] ou l'exemple sans diviseurs de zéro donné par R. A. Hirschfeld et $S$. Rolewicz [4]). Gh. Mocanu [7] a très légèrement étendu un des résultats de Le Page en prouvant que s'il existe $\alpha>0$ et une 
norme \|\|$_{1}$ telle que, sur $A$ unifère, on ait $\|x y\|_{1} \leqq \alpha\|y x\|$, alors $A$ est commutative.

Tous ces résultats qui précèdent vont être généralisés par les théorèmes suivants:

THÉORÈme 1. Soit A une algèbre de Banach complexe, avec unité. Les propriétés suivantes sont équivalentes:

$-1^{\circ} \quad A / \operatorname{Rad} A$ est commutative.

$-2^{\circ} \rho$ est uniformément continu sur $A$.

$-3^{\circ} i l$ existe un voisinage $V$ de l'unité et $k>0$ tels que $\mid \rho(x)-$ $\rho(y) \mid \leqq k\|x-y\|$, pour $x, y \in V$.

$-4^{\circ}$ il existe un voisinage $V$ de l'unité tel que $\rho(x+y) \leqq \rho(x)+$ $\rho(y)$, pour $x, y \in V$.

$-5^{\circ}$ il existe un voisinage $V$ de l'unité tel que $\rho(x y) \leqq \rho(x) \rho(y)$, pour $x, y \in V$.

$-6^{\circ}$ il existe $k>0$ tel que $\rho(x+y) \leqq k(\rho(x)+\rho(y))$, pour

$$
\rho(1-x)<1 \text { et } \rho(1-y)<1 \text {. }
$$

$-7^{\circ} \quad$ il existe $k>0$ tel que $\rho(x y) \leqq k \rho(x) \rho(y)$, pour $x, y \in A$.

$-8^{\circ} \quad \delta$ est uniformément continu sur $A$.

$-9^{\circ}$ il existe un voisinage $V$ de l'unité et $k>0$ tel que $\mid \delta(x)-$ $\delta(y) \mid \leqq k\|x-y\|$, pour $x, y \in V$.

$-10^{\circ} i l$ existe un voisinage $V$ de l'unité et $k>0$ tel que pour $x, y \in V$ on ait $\delta(x+y) \leqq k(\delta(x)+\delta(y))$.

ThÉorème 2. Soit A une algèbre de Banach complexe, sans unité. Les propritétés suivantes sont équivalentes:

$-1^{\circ} \quad A / \operatorname{Rad} A$ est commutative.

$-2^{\circ} \rho$ est uniformément continu sur $A$.

$-3^{\circ}$ il existe $k>0$ tel que $|\rho(x)-\rho(y)| \leqq k\|x-y\|$, pour $x, y \in A$.

$-4^{\circ}$ il existe $k>0$ tel que $\rho(x+y) \leqq k(\rho(x)+\rho(y))$, pour $x, y \in A$.

$-5^{\circ}$ il existe $k>0$ tel que $\rho(x y) \leqq k \rho(x) \rho(y)$, pour $x, y \in A$.

$-6^{\circ} \quad \delta$ est uniformément continu sur $A$.

$-7^{\circ}$ il existe $k>0$ tel que $|\delta(x)-\delta(y)| \leqq k\|x-y\|$, pour $x, y \in A$. $-8^{\circ}$ il existe $k>0$ tel que $\delta(x+y) \leqq k(\delta(x)+\delta(y))$, pour $x, y \in A$.

A l'origine, les démonstrations de ces deux théorèmes étaient élémentaires, mais très calculatoires. C'est ce travail, annoncé dans les Notices of the American Mathematical Society 18, p. 191 (1971), sous le titre "Almost commutative Banach algebras", qu'a utilisé D. Z. Spicer [9] pour donner une généralisation assez triviale de ces 
résultats. Depuis, l'utilisation des fonctions sous-harmoniques, dont le coup d'envoi a été donné par Vesentini [10], nous a permis de grandement simplifier ce travail et même, de l'améliorer fortement.

2. Quelques lemmes préliminaires. Soit $D$ un domaine de $C, \phi: D \rightarrow \boldsymbol{R} \cup\{-\infty\}$ est dite sous-harmonique si:

(a) $\phi$ est semi-continue supérieurement sur $D$, ce qui équivaut à dire que

$$
\varlimsup_{\substack{i \rightarrow i \\ i \neq i 0 \\ \lambda \in i j}} \dot{\phi}(\lambda) \leqq \dot{\phi}\left(\lambda_{0}\right)
$$

quel que soit $\lambda_{0} \in D$.

(b) $\oint$ possède la propriété de moyenne, c'est-à-dire que:

$$
\dot{\phi}\left(\lambda_{0}\right) \leqq \frac{1}{2 \pi} \int_{0}^{2 \pi} \phi\left(\lambda_{0}+r e^{i \theta}\right) d \theta,
$$

pour tout $\lambda_{0} \in D$ et $r>0$ tel que $\bar{B}\left(\lambda_{0}, r\right) \subset D$.

Rappelons quelques propriétés classiques de ces fonctions:

$-1^{\circ}$ Toute somme innie de fonctions sous-harmoniques est sousharmonique.

$-2^{\circ}$ Si $f$ est convexe et croissante et $\phi$ sous-harmonique alors $f \circ \phi$ est sous-harmonique.

$-3^{\circ}$ Si $\dot{\phi}_{1} \geqq \phi_{2} \geqq \cdots \geqq \phi_{n} \geqq \cdots$ est une suite décroissante de fonctions sous-harmoniques alors $\phi(\lambda)=\lim \dot{\phi}_{n}(\lambda)$ est sous-harmonique.

$-4^{\circ} \quad$ Si $\phi$ est sous-harmonique, $\phi \geqq 0$, telle que $\left|e^{\alpha \lambda}\right| \phi(\lambda)$ est sousharmonique pour tout $\alpha \in C$ alors Log $\dot{\varphi}$ est sous-harmonique.

$-5^{\circ} \mathrm{Si} \phi(\lambda, t)$ est une famille de fonctions sous-harmoniques en $\lambda$, intégrables par rapport à $t$ pour une mesure $\mu$ positive et finie, alors $\psi(\lambda)=\int \phi(\lambda, t) d \mu(t)$ est sous-harmonique.

$-6^{\circ}$ Si on désigne $M(r, \phi)=\operatorname{Max} \phi(\lambda)$, pour $|\lambda|=r$, et si

$$
\varliminf_{r \rightarrow \infty} \frac{M(r, \phi)}{\log r}=0
$$

alors $\phi$ est constante (analogue du théorème de Liouville).

$-7^{\circ}$ Théorème d'Oka-Rothstein: si $\dot{\phi}$ est sous-harmonique sur un domaine $D$ contenant 0 et si $\Gamma$ est un arc de Jordan contenu dans $D$, d'extrémité 0 alors:

$$
\phi(0)=\varlimsup_{\substack { \lambda \rightarrow 0 \\
\begin{subarray}{c}{\lambda \in 0 \\
j \neq 0{ \lambda \rightarrow 0 \\
\begin{subarray} { c } { \lambda \in 0 \\
j \neq 0 } }\end{subarray}} \phi(\lambda) .
$$

On pourra trouver les démonstration de ces résultats dans 


\section{Vladimirov [12].}

Lemme 1. Si $a \in A$ et si $U$ est un ouvert contenant $\operatorname{Sp} a$ alors il existe $r>0$ tel que $\|b-a\|<r$ implique $\operatorname{Sp} b \subset U$.

DÉmonstration. Voir Rickart [8], Théorème 1.6.16, p. 35-36.

CoRollaIRE. La fonction $x \rightarrow \delta(x)$ est semi-continue supérieurement sur $A$.

Lemme 2 (Vesentini [10] et [11]). Si $\lambda \rightarrow f(\lambda)$ est une fonction analytique d'un domaine $D$ de $C$ dans $A$ alors $\lambda \rightarrow \rho(f(\lambda))$ et $\lambda \rightarrow \log \rho(f(\lambda))$ sont sous-harmoniques.

DÉmonstration. (a ) Montrons d'abord que $\lambda \rightarrow \log \|f(\lambda)\|$ est sous-harmonique. C'est évident qu'elle est continue. D'après la formule de Cauchy pour les fonctions analytiques on a:

$$
f\left(\lambda_{0}\right)=\frac{1}{2 \pi} \int_{0}^{2 \pi} f\left(\lambda_{0}+r e^{i \theta}\right) d \theta
$$

donc:

$$
\left\|f\left(\lambda_{0}\right)\right\| \leqq \frac{1}{2 \pi} \int_{0}^{2 \pi}\left\|f\left(\lambda_{0}+r e^{i \theta}\right)\right\| d \theta
$$

D'après la propriété $4^{\circ}$ il suffit de prouver que $\lambda \rightarrow\left|e^{\alpha \lambda}\right|\|f(\lambda)\|$ est sous harmonique, mais c'est évident car $\left|e^{\alpha \lambda}\right|\|f(\lambda)\|=\left\|e^{\alpha \lambda} f(\lambda)\right\|$ et $\lambda \rightarrow e^{\alpha \lambda} f(\lambda)$ est analytique.

(b) Pour $x \in A$ on sait que la suite $\left\|x^{2^{n}}\right\|^{1 / 2^{n}}$ est décroissante et tend vers $\rho(x)$, donc d'après la propriété $3^{\circ}, \lambda \rightarrow \log \rho(f(\lambda))$ est sous-harmonique, puisque $\log \rho(f(\lambda))=\lim _{n \rightarrow \infty} 1 / 2^{n} \log \left\|f(\lambda)^{2^{n}}\right\|$, où $\lambda \rightarrow f(\lambda)^{2^{n}}$ est analytique.

(c) La fonction $t \rightarrow e^{t}$ est convexe et croissante, donc, d'après la propriété $2^{\circ}, \lambda \rightarrow \rho(f(\lambda))$ est sous-harmonique.

Lemme 3. Pour $x \in A$, soit $\gamma(x)=1 / 2 \pi \int_{0}^{2 \pi} \log \rho\left(\exp \left(e^{i \theta} x\right)\right) d \theta$. Elle possède les propriétés suivantes:

$-1^{\circ} \quad 0 \leqq \gamma(x) \leqq \rho(x)$, quel que soit $x \in A$.

$-2^{\circ} \quad \gamma(x)=0$ implique que le spectre de $x$ a un seul point.

$-3^{\circ} \quad$ Si $\lambda \rightarrow f(\lambda)$ est une fonction analytique d'un domaine $D$ dans $A$, alors $\lambda \rightarrow \gamma(f(\lambda))$ est sous-harmonique.

\section{DEMONSTRATION.}

$-1^{\circ}$ Comme $1 \leqq \rho\left(\exp \left(e^{i \theta} x\right)\right) \rho\left(\exp \left(e^{i(\theta+\pi)} x\right)\right)$ il est immédiat que 
$\gamma(x) \geqq 0$. l'autre inégalité résulte du fait que $\rho\left(\exp \left(e^{i \theta} x\right)\right) \leqq$ $\exp \left(\rho\left(e^{i \theta} x\right)\right)=\exp \rho(x)$.

$-2^{\circ}$ Soit $\psi(\theta)=\log \rho\left(\exp \left(e^{i \theta} x\right)\right)+\log \rho\left(\exp \left(-e^{i \theta} x\right)\right) \geqq 0$.

Cette fonction est continue, puisque $\rho$ est continu sur la sous-algèbre commutative engendrée par $x$ et l'on a:

$$
\gamma(x)=\frac{1}{2 \pi} \int_{0}^{\pi} \psi(\theta) d \theta
$$

Donc si $\gamma(x)=0$ alors $\psi(\theta) \equiv 0$, pour $0 \leqq \theta \leqq \pi$, ce qui implique que $\operatorname{Sp}\left(\exp \left(e^{i \theta} x\right)\right)$ est contenu dans un cercle centré en 0 , quel que soit $\theta$, donc pour $\lambda, \mu \in \operatorname{Spx}$ on a $\operatorname{Re} e^{i \theta}(\lambda-\mu)=0$ quel soit $\theta$, soit $\lambda=\mu$.

$-3^{\circ}$ Cela résulte immédiatement du Lemme 2 et de la propriété 5 .

Lemme 4. Si quels que soient $x, y \in A, \operatorname{Sp}(x y=y x)$ a un seul point, alors $A / \operatorname{Rad} A$ est commutative.

DÉmonstration. Soit $\pi$ une représentation irréductible sur un espace de Banach $E$. Si $\operatorname{dim} E=1$, quel que soit $\pi$, c'est terminé. Supposons donc $\operatorname{dim} E>1$. D'après le théorème de transitivité des algèbres irréductibles, pour $\xi, \eta$ indépendants dans $E$, il existe $x, y \in A$ tels que:

$$
\begin{array}{ll}
\pi(x) \xi=\eta & \pi(y) \xi=0 \\
\pi(x) \eta=0 & \pi(y) \eta=\xi
\end{array}
$$

alors

$$
\pi(x y-y x) \xi=-\xi \text { et } \pi(x y-y x)=\eta
$$

donc $\{-1,1\} \subset \operatorname{Sp} \pi(x y-y x) \subset \operatorname{Sp}(x y-y x)$, ce qui est absurde.

Lemme 5. Si | | est une semi-norme sur A, telle que $|x| \leqq \rho(x)$, quel que soit $x \in A$, alors $|x y-y x|=0$, quels que soient $x, y \in A$.

Démonstration. (a) Soit $\lambda \rightarrow f(\lambda)$ une fonction holomorphe de $D$ dans $A$, alors $\lambda \rightarrow|f(\lambda)|$ est continue car:

$$
\begin{aligned}
\|f(\lambda)|-| f(\mu)\| & \leqq|f(\lambda)-f(\mu)| \leqq \rho(f(\lambda)-f(\mu)) \\
& \leqq\|f(\lambda)-f(\mu)\|
\end{aligned}
$$

Elle est sous-harmonique, car évidemment:

$$
\left|f\left(\lambda_{0}\right)\right| \leqq \frac{1}{2 \pi} \int_{0}^{2 \pi}\left|f\left(\lambda_{0}+r e^{i \theta}\right)\right| d \theta
$$


(b) Soient $x, y \in A$ et $f(\lambda)=e^{\lambda x} y e^{-\lambda x}$. C'est une fonction holomorphe de $C$ dans $A$, même si $A$ n'a pas d'unité, car

$$
e^{\lambda x} y e^{-\lambda x}=y+\lambda[x, y]+\frac{\lambda^{2}}{2 !}[x,[x, y]]+\cdots \in A .
$$

Aussi

$$
\left|y+\lambda[x, y]+\frac{\lambda^{2}}{2 !}[x,[x, y]]+\cdots\right| \leqq \rho\left(e^{\lambda x} y e^{-\lambda x}\right)=\rho(y)
$$

done

$$
\left|[x, y]+\frac{\lambda}{2}[x,[x, y]]+\cdots\right| \leqq \frac{|y|+\rho(y)}{|\lambda|}
$$

Mais $\lambda \rightarrow|[x, y]+\lambda / 2[x,[x, y]]+\cdots|$ est sous-harmonique, d'après ce qui précède, et tend vers 0 quand $\lambda$ tend vers l'infini, donc cette fonction est identiquement nulle, soit $|[x, y]|=0$.

Ce lemme est une généralisation du résultat de Hirschfeld et Żelazko. L'énoncé qui suit est un cas particulier de la formule de Trotter.

Lemme 6. Supposons $A$ avec unité et $x, y \in A$. On $a$ :

$$
e^{x+y}=\lim _{\substack{\lambda \rightarrow 0 \\ \lambda \neq 0}}\left(e^{\lambda x} \cdot e^{\lambda y}\right)^{1 / \lambda}
$$

et il existe une suite $\left(\lambda_{n}\right)$ tendant vers 0 , avec $\lambda_{n}>0$, telle que:

$$
\rho\left(e^{x+y}\right)=\lim _{n \rightarrow \infty} \rho\left(e^{\lambda_{n} x} \cdot e^{\lambda_{n} y}\right)^{1 / \lambda_{n}} .
$$

DÉmonstration. Il existe $r>0$ tel que si $|\lambda| \leqq r$ also

$$
\left\|e^{\lambda x} \cdot e^{\lambda y}-1\right\|<1,
$$

donc $\log \left(e^{\lambda x} \cdot e^{\lambda y}\right)$ est alors parfaitement défini. Considérons la fonction définie par:

$$
\psi(y)=\exp \left(\frac{1}{\lambda} \log \left(e^{\lambda x} \cdot e^{\lambda y}\right)\right) \text { si } 0<|\lambda| \leqq r \text { et } \psi(0)=e^{x+y} .
$$

Il n'est pas difficile de voir que cette fonction est analytique pour $|\lambda| \leqq r$. D'après le théorème d'Oka-Rothstein, si on prend $\Gamma=] 0, r]$, il existe une suite $\left(\lambda_{n}\right), \lambda_{n} \in \Gamma$ telle que:

$$
\rho\left(e^{x+y}\right)=\lim \rho\left(\left(e^{\lambda_{n} x} \cdot e^{\lambda_{n} y}\right)^{1 / \lambda_{n}}\right) .
$$

Mais si $\log a$ est défini et $\mu>0$ on a $\rho\left(a^{\mu}\right)=\rho\left(e^{\mu \log a}\right)=\rho(a)^{\mu}$ car 
c'est vrai pour $\mu$ rationnel et il suffit d'étendre le résultat par continuité, puisque $\rho$ est continu sur la sous-algèbre avec unité engendrée par $a$.

Lemme 7. Quel que soit $x \in A$, le diamètre du spectre de $x$ vérifie

$$
\delta(x)=\operatorname{Max}_{|\alpha|=1}\left(\log \rho\left(e^{\alpha x}\right)+\log \rho\left(e^{-\alpha x}\right)\right)
$$

Si $\lambda \rightarrow f(\lambda)$ est une fonction analytique de $D$ dans $A$ alors $\lambda \rightarrow$ Log $\delta(f(\lambda))$ est sous-harmonique.

DÉmonstration. ( a ) Soient $\lambda_{1}, \lambda_{2} \in \operatorname{Sp} x$, tels que $\left|\lambda_{1}-\lambda_{2}\right|=\delta(x)$. Alors $\log \rho\left(e^{\alpha x}\right)+\log \rho\left(e^{-\alpha x}\right) \geqq \log \left|e^{\alpha \lambda_{1}}\right|+\log \left|e^{-\alpha \lambda_{2}}\right|=\operatorname{Re}\left(\alpha\left(\lambda_{1}-\lambda_{2}\right)\right)$, donc en prenant $\alpha=e^{-i \theta}$, où $\theta=\operatorname{Arg}\left(\lambda_{1}-\lambda_{2}\right)$ on obtient:

$$
\delta(x)=\left|\lambda_{1}-\lambda_{2}\right| \leqq \operatorname{Max}_{|\alpha|=1}\left(\log \rho\left(e^{\alpha x}\right)+\log \rho\left(e^{-\alpha x}\right)\right) .
$$

Dans l'autre sens, la fonction $\alpha \rightarrow \log \rho\left(e^{\alpha x}\right)+\log \rho\left(e^{-\alpha x}\right)$ est semicontinue supérieurement, donc atteint son maximum $\operatorname{sur}\{z|| z \mid=1\}$ en un point $\alpha_{0}$. Choisissons $\lambda_{1}$ et $\lambda_{2}$ de façon que $\left|e^{\alpha_{0} \lambda_{1}}\right|=\rho\left(e^{\alpha_{0} x}\right)$ et $\left|e^{-\alpha_{0} \lambda_{2}}\right|=\rho\left(e^{-\alpha_{0} x}\right)$. Alors

$$
\begin{aligned}
\operatorname{Max}_{|\alpha|=1}\left(\log \rho\left(e^{\alpha x}\right)+\log \rho\left(e^{-\alpha x}\right)\right) & =\operatorname{Re}\left(\alpha_{0}\left(\lambda_{1}-\lambda_{2}\right)\right) \\
& \leqq\left|\lambda_{1}-\lambda_{2}\right| \leqq \delta(x) .
\end{aligned}
$$

(b ) Pour $\alpha$ fixé, $\phi_{\alpha}(\lambda)=\log \rho(\exp (\alpha f(\lambda)))+\log \rho(\exp (-\alpha f(\lambda)))$ est sous-harmonique, d'après le Lemme 2. D'où:

$$
\begin{aligned}
\operatorname{Max}_{|\alpha|=1} \phi_{\alpha}\left(\lambda_{0}\right) & \leqq \frac{1}{2 \pi} \operatorname{Max}_{|\alpha|=1} \int_{0}^{2 \pi} \phi_{\alpha}\left(\lambda_{0}+r e^{i \theta}\right) d \theta \\
& \leqq \frac{1}{2 \pi} \int_{0}^{2 \pi} \operatorname{Max}_{|\alpha|=1} \phi_{\alpha}\left(\lambda_{0}+r e^{i \theta}\right) d \theta
\end{aligned}
$$

donc $\lambda \rightarrow \delta(f(\lambda))$ possède la propriété de moyenne, mais la semicontinuité supérieure résulte du corollaire du Lemme 1.

(c) Si on remarque que $\left|e^{\alpha \lambda}\right| \delta(f(\lambda))=\delta\left(e^{\alpha \lambda} f(\lambda)\right)$ on conclut que $\lambda \rightarrow \log \delta(f(\lambda))$ est sous-harmonique, d'après la propriété $4^{\circ}$.

3. Démonstrations des théorèmes. D'abord, donnons celle du premier théorème.

$1^{\circ} \Rightarrow n^{\circ}, 2 \leqq n \leqq 10$. Comme $\operatorname{Sp} x=\operatorname{Sp} \dot{x}$, où $\dot{x}$ est la classe de $x$ dans $A / \operatorname{Rad} A$ il est clair que

$$
\begin{aligned}
& \rho(x+y)=\rho(\dot{x}+\grave{y}) \leqq \rho(\dot{x})+\rho(\grave{y})=\rho(x)+\rho(y) \\
& \rho(x y)=\rho(\dot{x} \dot{y}) \leqq \rho(\dot{x}) \rho(\dot{y})=\rho(x) \rho(y) \\
& |\rho(x)-\rho(y)| \leqq \rho(x-y) \leqq\|x-y\| .
\end{aligned}
$$


De plus $\delta(x)=\delta(\dot{x})=\operatorname{Max}|\chi(\stackrel{\circ}{x})-\eta(\stackrel{\circ}{x})|$, pour tous les $\chi, \eta$ caractères de $A / \operatorname{Rad} A$ donc:

$$
\begin{aligned}
\delta(x+y) & \leqq \delta(x)+\delta(y) \\
|\delta(x)-\delta(y)| & \leqq \delta(x-y) \leqq \rho(x-y) \leqq\|x-y\| .
\end{aligned}
$$

$2^{\circ} \Rightarrow 3^{\circ}$. Soit $\varepsilon>0$, il existe $\alpha>0$ tel que $\|x-y\|<\alpha$ implique $|\rho(x)-\rho(y)|<\varepsilon$. Soient $x \neq y$ quelconques, prenons $a=\alpha x /(2\|x-y\|)$ et $b=\alpha y /(2\|x-y\|)$, alors $\|a-b\|<\alpha$, donc

$$
|\rho(x)-\rho(y)|<\frac{2 \varepsilon}{\alpha}\|x-y\|
$$

pour $x, y \in A$.

$3^{\circ} \Rightarrow 1^{\circ}$. Soient $x, y \in A$ et $M$ tel que $\|x\|,\|y\| \leqq M$. Choisissons $r j>0$ de façon que $e^{\lambda x}$, $e^{\lambda y} \in V$ et $\rho\left(e^{\lambda x}\right), \rho\left(e^{\lambda x}\right) \geqq 1 / 2$ pour $|\lambda| \leqq r$, ce qui est possible car $\rho$ est continu sur la sous-algèbre commutative, avec unité, engendrée par un élément.

Alors

$$
\begin{aligned}
& \left|\log \rho\left(e^{\lambda x}\right)-\log \rho\left(e^{\lambda y}\right)\right| \\
& \quad \leqq 2\left|\rho\left(e^{\lambda x}\right)-\rho\left(e^{\lambda y}\right)\right| \leqq 2 k\left\|e^{\lambda x}-e^{\lambda y}\right\|
\end{aligned}
$$

done

$$
\left|\log \rho\left(e^{\lambda x}\right)-\log \rho\left(e^{\lambda y}\right)\right| \leqq 2 k r\|x-y\|+4 k \sum_{n=2}^{\infty} \frac{(M r)^{n}}{n !}
$$

Posons $\gamma_{r}(x)=1 /(2 \pi r) \int_{0}^{2 \pi} \log \rho\left(\exp \left(r e^{i \theta} x\right)\right) d \theta$. D'après la définition de $\gamma$ donnée dans le Lemme 3, il n'est pas difficile de voir que $\gamma(x)=$ $\gamma_{r}(x)$ donc que:

$$
|\gamma(x)-\gamma(y)| \leqq 2 k\|x-y\|+4 k r e^{M} \quad \text { si } \quad 0<r \leqq 1 .
$$

En faisant tendre $r$ vers 0 on obtient $|\gamma(x)-\gamma(y)| \leqq 2 k\|x-y\|$ pour $x, y \in A$.

Prenons $x=e^{\lambda a} b e^{-\lambda a}$ et $y=\lambda[a, b]+\lambda^{2} / 2 ![a,[a, b]]+\cdots$, alors d'après ce qui précède $\gamma(y) \leqq \gamma\left(e^{\lambda a} b e^{-\lambda a}\right)+2 k\|b\| \leqq(2 k+1)\|b\|$ puisque $\gamma\left(e^{\lambda a} b e^{-\lambda a}\right) \leqq \rho\left(e^{\lambda a} b e^{-\lambda a}\right)=\rho(b) \leqq\|b\|$. Ainsi

$$
\gamma\left([a, b]+\frac{\lambda}{2 !}[a,[a, b]]+\frac{\lambda^{2}}{3 !}[a,[a,[a, b]]]+\cdots\right) \leqq \frac{(2 k+1)\|b\|}{|\lambda|}
$$

En appliquant le Lemme 3 et la propriété $6^{\circ}$ on obtient que $\gamma(a b-b a)=0$ quels que soient $a, b \in A$, donc que $\operatorname{Sp}(a b-b a)$ a un seul point, mais alors d'après le Lemme $4, A / \operatorname{Rad} A$ est commutative. $4^{\circ} \Rightarrow 1^{\circ}$ Posons $\theta(x)=\varlimsup_{\substack{\lambda \rightarrow 0 \\ \lambda \neq 0}}(|\rho(1+\lambda x)-1|) /|\lambda|$. C'est évident que $0 \leqq \theta(x) \leqq \rho(x)$. 
De plus

$$
\theta(\alpha x)=\varlimsup_{\substack{\lambda, \alpha \rightarrow 0 \\ \lambda \alpha \neq 0}} \frac{|\rho(1+\lambda \alpha x)-1|}{|\lambda \alpha|} \cdot|\alpha|=|\alpha| \theta(x),
$$

pour $\alpha \neq 0$ et $\theta(0)=0$. Montrons maintenant que $\theta(x+y) \leqq \theta(x)+$ $\theta(y)$. Pour $\lambda$ assez petit, $1+\lambda x, 1+\lambda y$ et $1+\lambda(x+y) / 2$ sont dans $V$, donc:

$$
\begin{gathered}
\rho(2+\lambda(x+y)) \leqq \rho(1+\lambda x)+\rho(1+\lambda y), \text { soit, } \\
\theta\left(\frac{x+y}{2}\right) \leqq \frac{1}{2}(\theta(x)+\theta(y)),
\end{gathered}
$$

qui avec ce qui précède, donne $\theta(x+y)=2 \theta((x+y) / 2) \leqq \theta(x)+\theta(y)$. D'après le Lemme 5 , on a donc $\theta(x y-y x)=0$, quels que soient $x, y \in A$. Supposons que $\theta(\alpha)=0$ et soit $\alpha+i \beta \in \operatorname{Sp} \alpha$, alors $1+\lambda \alpha+$ $\lambda i \beta \in \operatorname{Sp}(1+\lambda a)$ donc $\rho(1+\lambda a) \geqq\left((1+\lambda \alpha)^{2}+\lambda^{2} \beta^{2}\right)^{1 / 2}$ alors

$$
\frac{\left((1+\lambda \alpha)^{2}+\lambda^{2} \beta^{2}\right)^{1 / 2}-1}{\lambda} \longrightarrow 0
$$

quand $\lambda \rightarrow 0$, ce qui implique $\alpha=0$, soit $\operatorname{Sp} \alpha \subset i \boldsymbol{R}$; mais on a également $\theta(i a)=0$ donc $\operatorname{Sp} a \subset \boldsymbol{R}$, soit $\rho(a)=0$.

Ainsi, d'après le Lemme 4, puisque $\operatorname{Sp}(x y-y x)=\{0\}$, quels que soient $x, y \in A$, alors $A / \operatorname{Rad} A$ est commutative.

$5^{\circ} \Rightarrow 1^{\circ}$. Posons $\nu(x)=\log \rho\left(e^{x}\right)$, montrons que

$$
\nu(x+y) \leqq \nu(x)+\nu(y) .
$$

D'après le Lemme 6 , il existe une suite $\left(\lambda_{n}\right)$ tendant vers 0 , avec $\lambda_{n}>0$, telle que $\rho\left(e^{x+y}\right)=\lim _{n \rightarrow \infty} \rho\left(e^{\lambda_{n} x} \cdot e^{\lambda_{n} y}\right)^{1 / \lambda}$. Mais pour $\lambda_{n}$ assez petit $e^{\lambda_{n} x}$ et $e^{\lambda_{n} y}$ sont dans $V$, donc on $a$ :

$$
\rho\left(e^{x+y}\right) \leqq \varlimsup_{n \rightarrow \infty}\left[\rho\left(e^{\lambda_{n} x}\right) \cdot \rho\left(e^{\lambda_{n} y}\right)\right]^{1 / \lambda_{n}}=\rho\left(e^{x}\right) \cdot \rho\left(e^{y}\right),
$$

d'où le résultat.

D'une façon identique on obtient $\nu(-(x+y)) \leqq \nu(-x)+\nu(-y)$, d'où puisque $\delta(x)=\operatorname{Max}_{|\alpha|=1}(\nu(\alpha x)+\nu(-\alpha x))$, on $a$ :

$$
\delta(x+y) \leqq \delta(x)+\delta(y) .
$$

Comme il est évident que $0 \leqq \delta(x) \leqq \rho(x)$ et que $\delta(\alpha x)=|\alpha| \delta(x)$ pour $\alpha \in C$ et $x \in A$, d'après les Lemmes 4 et $5, A / \operatorname{Rad} A$ est commutative.

$6^{\circ} \Rightarrow 1^{\circ}$. Supposons que $\rho(b-1)<1$ et que $a \in A$. Alors

$$
\rho\left(e^{\lambda a} b e^{-\lambda a}-1\right)=\rho\left(e^{\lambda a}(b-1) e^{-\lambda a}\right)=\rho(b-1)<1,
$$

donc $\rho\left(\lambda[a, b]+\lambda^{2} / 2 ![a,[a, b]]+\cdots\right) \leqq k\left(\rho(b-1)+\rho\left(e^{\lambda a} b e^{-\lambda a}-1\right)\right)$ 
soit

$$
\rho\left([a, b]+\frac{\lambda}{2 !}[a,[a, b]]+\cdots\right) \leqq \frac{2 k \rho(b-1)}{|\lambda|}
$$

D'après la propriété $6^{\circ}$, on obtient $\rho(a b-b a)=0$. Maintenant si $b$ est quelconque, pour $\lambda$ assez petit $\rho((1+\lambda b)-1)<1$, d'où d'après ce qui précède $\rho((1+\lambda b) a-a(1+\lambda b))=|\lambda| \rho(a b-b a)=0$, ainsi, d'après le Lemme $4, A / \operatorname{Rad} A$ est commutative.

$7^{\circ} \Rightarrow 3^{\circ}$. Montrons d'abord que $d(\lambda, \operatorname{Sp} y) \leqq k\|x-y\|$, si $\lambda \in \operatorname{Sp} x$, où $d(\lambda, \operatorname{Sp} y)=\operatorname{Inf}|\lambda-\mu|$, pour $\mu \in \operatorname{Sp} y$. Supposons que pour un $\lambda \in \operatorname{Sp} x$ on ait $d(\lambda, \operatorname{Sp} y)>k\|x-y\|$, alors $\lambda-y$ est inversible et $\lambda-x=(\lambda-y)\left[1+(\lambda-y)^{-1}(y-x)\right]$, mais:

$$
\begin{aligned}
\rho\left((\lambda-y)^{-1}(y-x)\right) & \leqq k \rho\left((\lambda-y)^{-1}\right) \rho(y-x) \\
& =\frac{k \rho(y-x)}{d(\lambda, \operatorname{Sp} y)}<\frac{\rho(y-x)}{\|y-x\|}<1
\end{aligned}
$$

donc, $\lambda-x$ est inversible, ce qui est absurde. D'une façon identique, on prouve que $d(\mu, \operatorname{Sp} x) \leqq k\|x-y\|$, pour $\mu \in \operatorname{Sp} y$, donc:

$$
|\rho(x)-\rho(y)| \leqq k\|x-y\| \text {. }
$$

$8^{\circ} \Rightarrow 9^{\circ}$. Démonstration identique à $2^{\circ} \Rightarrow 3^{\circ}$.

$9^{\circ} \Rightarrow 1^{\circ}$. Comme $\operatorname{Sp}(1+x)=1+\operatorname{Sp} x$, on $a \delta(1+\lambda x)=|\lambda| \delta(x)$ quels que soient $\lambda \in C$ et $x \in A$. Si $x, y \in A$, pour $\lambda$ assez petit, on a $1+\lambda x \in V$ et $1+\lambda y \in V$ donc.

$$
|\lambda||\delta(x)-\delta(y)|=|\delta(1+\lambda x)-\delta(1+\lambda y)| \leqq k|\lambda|\|x-y\|,
$$

soit $|\delta(x)-\delta(y)| \leqq k\|x-y\|$, quels que soient $x, y \in A$.

En reprenant, avec $\delta$, la fin de la démonstration de $3^{\circ} \Rightarrow 1^{\circ}$, on obtient que $\delta(a b-b a)=0$, quels que soient $a, b \in A$ donc que $\operatorname{Sp}(a b-b a)$ a un seul point, mais alors d'après le Lemme $4, A / \operatorname{Rad} A$ est commutative.

$10^{\circ} \Rightarrow 1^{\circ}$. Soient $x, y \in A$, pour $\lambda$ assez petit, $1+\lambda x, 1+\lambda y$ sont dans $V$, donc $\delta(2+\lambda(x+y))=|\lambda| \delta(x+y) \leqq k|\lambda|(\delta(x)+\delta(y))$, d'où $\delta(x+y) \leqq k(\delta(x)+\delta(y))$ quels que soient $x, y \in A$. On obtient comme plus haut que:

$$
\delta\left([a, b]+\frac{\lambda}{2 !}[a,[a, b]]+\cdots\right) \leqq \frac{2 \delta(b)}{|\lambda|}
$$

donc, d'après les Lemmes 4 et 5 que $A / \operatorname{Rad} A$ est commutative.

Pour le deuxième théorème, la démonstration est identique. Il suffit seulement de remarquer que, si $a, b \in A$, alors $e^{\lambda a}$ n'est pas défini 
dans $A$, mais, par contre, $e^{\lambda a} b e^{-\lambda a}=b+\lambda[a, b]+\lambda^{2} / 2 ![a,[a, b]]+\cdots$ l'est.

Remarque 1. Dans $M_{n}(C)$, considérons $V=\{x|\cdot| \mid x-1 \| \leqq 1 / 2\}$. C'est un voisinage compact de 1 , de plus la fonction $x \rightarrow \rho(x) /\|x\|$ est continue sur $V$, donc atteint sa borne inférieure $\gamma \geqq 0$. Si $\gamma=0$, alors il existe $a \in V$, tel que $\rho(\alpha)=0$, ce qui est absurde car:

$$
1=\rho(1) \leqq \rho(a)+\rho(1-a) \leqq\|1-a\| \leqq \frac{1}{2} .
$$

Donc $\rho(x) \geqq \gamma\|x\|$, sur $V$, ce qui implique que pour $x, y \in V$ on ait $\rho(x+y) \leqq\|x+y\| \leqq\|x\|+\|y\| \leqq 1 / \gamma(\rho(x)+\rho(y))$ et

$$
\rho(x y) \leqq\|x y\| \leqq\|x\|\|y\| \leqq \frac{1}{\gamma^{2}} \rho(x) \rho(y) .
$$

Cet exemple prouve que, dans les propriétés $4^{\circ}$ et $5^{\circ}$ du Théorème 1 , on ne peut pas avoir $\rho(x+y) \leqq k(\rho(x)+\rho(y))$ ou $\rho(x y) \leqq k \rho(x) \rho(y)$. Nécessairement il faut $k=1$.

4. Quelques corollaires. Une algèbre avec involution est dite symétrique si, pour tout élément hermitien $h$ (c'est-à-dire vérifiant $h=h^{*}$ ), on a $\operatorname{Sp} h \subset \boldsymbol{R}$. V. Pták (voir [2]) a prouvé qu'en posant $|x|=\rho\left(x^{*} x\right)^{1 / 2},|x|$ est une semi-norme sous-multiplicative sur $A$, telle que $|x| \geqq \rho(x)$ pour $x \in A$ et $|x|=\rho(x)$ pour $x$ normal (c'est-à-dire vérifiant $\left.x^{*} x=x x^{*}\right)$.

Corollaire 1. A/Rad $A$ est une algèbre symétrique commutative si et seulement si $\rho\left(x^{*} x\right)=\rho(x)^{2}$, quel que soit $x \in A$.

DÉmonstration. La condition nécessaire est évidente. Réciproquement supposons que $\rho\left(x^{*} x\right)=\rho(x)^{2}$, quel que soit $x \in A$. Si $A$ a une unité, prenons $x=e^{i h}$, pour $h$ hermitien et alors $\rho\left(e^{i h}\right)^{2}=$ $\rho\left(e^{-i h} e^{i h}\right)=1$. De la même façon $\rho\left(e^{-i h}\right)=1$. Ces deux résultats prouvent que $\operatorname{Sp} e^{i h}$ est contenu dans $\{z|| z \mid=1\}$, donc que $\operatorname{Sp} h \subset \boldsymbol{R}$, d'où $A$ est symétrique. Si $A$ n'a pas d'unité c'est un peu plus difficile de prouver qu'elle est symétrique. Supposons qu'il existe $h$ hermitien tel que $\operatorname{Sp} h \not \subset \boldsymbol{R}$; on peut supposer que $\alpha+i \in \operatorname{Sp} h$. Soit $B$ une sous-algèbre, fermée, involutive, commutative, maximale, contenant $h$; on sait alors que $\operatorname{Sp}_{A} x=\operatorname{Sp}_{B} x$, quel que soit $x \in B$. Posons $\quad v=(h-\alpha+n i)^{m} h \in B$. Comme $\alpha+i \in \operatorname{Sp}_{A} h=\operatorname{Sp}_{B} h$, il existe un caractère $\chi$ de $B$ tel que $\chi(h)=\alpha+i \neq 0$. Alors $\chi(v)=(n+1)^{m} i^{m}(\alpha+i)$ donc $\rho(v) \geqq(n+1)^{m}\left(1+\alpha^{2}\right)^{1 / 2}$. Mais $v^{*} v=$ $\left((h-\alpha)^{2}+n^{2}\right)^{m} h^{2}$ donc $\rho\left(v^{*} v\right) \leqq \rho(h)^{2}\left[(\rho(h)+|\alpha|)^{2}+n^{2}\right]^{m}$ on obtient ainsi $(n+1)^{2 m}\left(1+\alpha^{2}\right) \leqq \rho(h)^{2}\left[(\rho(h)+|\alpha|)^{2}+n^{2}\right]^{m}$, d'où: 


$$
(n+1)^{2}\left(1+\alpha^{2}\right)^{1 / m} \leqq \rho(h)^{2 / m}\left[(\rho(h)+|\alpha|)^{2}+n^{2}\right]
$$

soit en faisant tendre $m$ vers l'infini $(n+1)^{2} \leqq(\rho(h)+|\alpha|)^{2}+n^{2}$, ce qui est absurde pour $2 n+1>(\rho(h)+|\alpha|)^{2}$. Sachant maintenant qu'elle est symétrique, alors d'après Pták $\rho(x)=|x|$, où $\mid$ | est une semi-norme, donc d'après le Théorème $1, A / \operatorname{Rad} A$ est commutative.

Remarque 2. Dans le cas où $A$ a une unité, on est capable de prouver beaucoup mieux: $A / \operatorname{Rad} A$ est symétrique et commutative si et seulement si $\rho\left(x^{*} x\right)=\rho(x)^{2}$ dans un voisinage $V$ de l'unité.

La démonstration de la symétrie se fait de la même façon, sauf qu'on remplace $h$ par $\lambda h$ avec $\lambda \in \boldsymbol{R}$, assez petit pour que $e^{i \lambda h} \in V$. Ainsi, d'après Pták, on a $|x| \geqq \rho(x)$ quel que soit $x$ et $|x|=\rho(x)$ pour $x \in V$. Raisonnons dans $A^{\prime}=A / \operatorname{Rad} A$, où $|x|=0$ implique $x=0$. Soit $x \in A^{\prime}$ alors $e^{x / 2^{n}} \in V$, pour $n$ assez grand donc:

$$
\left|e^{x}\right| \leqq\left|e^{x / 2}\right|^{2} \leqq \cdots \leqq\left|e^{x / 2^{n}}\right|^{2^{n}}=\rho\left(e^{x / 2^{n}}\right)^{2^{n}}=\rho\left(e^{x}\right) \leqq\left|e^{x}\right|
$$

soit $\left|e^{x}\right|=\rho\left(e^{x}\right)$, que que soit $x$.

Posons $k=\operatorname{Inf}_{x \neq 0} \rho(x) /|x| \leqq 1$ et choisissons une suite $\left(x_{n}\right)$ telle que $\rho\left(x_{n}\right) \leqq(k+1 / n)\left|x_{n}\right|$, quitte à multiplier les $x_{n}$ par une constante on peut aussi supposer que $\left|x_{n}\right|=1 /(k+2 / n)$. Alors

$$
\rho\left(x_{n}\right) \leqq \frac{k+1 / n}{k+2 / n}<1
$$

donc, il existe $u_{n}$ tel que $1+x_{n}=e^{u_{n}}$, mais alors d'après ce qui précède $\rho\left(1+x_{n}\right)=\left|1+x_{n}\right|$ et de plus, si on suppose $k<1 / 3$, alors $\left|x_{n}\right|>1$, à partir d'un certain rang, donc:

$$
\left|x_{n}\right|-1=\frac{1}{k+2 / n}-1<\left|1+x_{n}\right|=\rho\left(1+x_{n}\right) \leqq 1+\frac{k+1 / n}{k+2 / n},
$$

soit $k \geqq 1 / 3$, d'où absurdité. Ainsi $\rho(x) \geqq 1 / 3|x|$ quel que soit $x \in A^{\prime}$, d'où, d'après le Théorème, $1, A / \operatorname{Rad} A$ est commutative.

Dans [1], nous avons démontré que si $\rho$ est sous-multiplicatif sur l'ensemble des éléments normaux de $A$ involutive alors le spectre est uniformément continu sur cet ensemble. C'est la méthode de démonstration que nous exploiterons pour prouver le:

Corollaire 2. Supposons que $A$ est une algèbre involutive vérifiant:

$1^{\circ}$ quel que soit $x \in A$ on a $\rho\left(\left(x+x^{*}\right) / 2\right) \leqq \rho(x)$.

$2^{\circ}$ il existe $\alpha>0$ tel que $\rho(x y) \leqq \alpha \rho(x) \rho(y)$, pour $x, y$ normaux. Alors $A / \operatorname{Rad} A$ est commutative. 
DÉmonstration. Nous donnerons les grandes idées, dans le cas avec unité, en priant le lecteur de se reporter à [1] pour la démonstration des propriétés que nous énoncerons.

Posons $|x|=\alpha \operatorname{Inf} \sum\left|\lambda_{i}\right| \rho\left(u_{i}\right)$, pour toutes les décompositions finies $x=\sum \lambda_{i} \cdot u_{i}+v$, où $\lambda_{i} \in C, u_{i}$ unitaire et $v \in \operatorname{Rad} A$. On vérifie que c'est une semi-norme sur $A$ telles que $|h| \leqq \alpha(1+\sqrt{2}) \rho(h)$ si $h$ est hermitien, donc pour $x \in A$ on a, d'après l'hypothèse $1^{\circ}$ :

$$
\begin{aligned}
|x| & \leqq\left|\frac{x+x^{*}}{2}\right|+\left|\frac{x-x^{*}}{2}\right| \leqq \alpha(1+\sqrt{2})\left(\rho\left(\frac{x+x^{*}}{2}\right)+\rho\left(\frac{x-x^{*}}{2 i}\right)\right) \\
& \leqq 2 \alpha(1+\sqrt{2}) \rho(x) .
\end{aligned}
$$

En appliquant le Lemme 5, on conclut que $|a b-b a|=0$, quels que soient $a, b \in A$. Mais, dans [1], après quelques calculs on peut obtenir que $|x| \geqq \rho(x)$, quel que soit $x \in A$, ainsi $\rho(a b-b a)=0$, soit en appliquant le Lemme $4, A / \operatorname{Rad} A$ est commutative.

Additif sur épreuves (30/4/76). Depuis, nous avons résolu négativement la seconde conjecture de Hirschfeld et Z̊elazko.

\section{REFERENCES}

1. B. Aupatit, Uniforme continuité du spectre dans les algèbres de Banach avec involution, à paraître.

2. A. F. Bonsall and J. Duncan, Complete normed algebras, New-York, Springer-Verlag, 1973.

3. J. Duncan and A. W. Tullo, Finite dimensionality, nilpotents and quasinilpotents in Banach algebras, Proc. Edinburgh Math. Soc., 19 (1974), 45-48.

4. R. A. Hirschfeld, and S. Rolewicz, A class of non-commutative Banach algebras without divisors of zero, Bull. Acad. Polon. Sci., 17 (1969), 751-753.

5. R. A. Hirschfeld and W. Żelazko, On spectral norm Banach algebras, Bull. Acad. Polon. Sci., 16 (1968), 195-199.

6. C. Le Page, Sur quelques conditions entraînant la commutativité dans les algèbres de Banach, C. R. Acad. Sci. Paris, 265 (1967), 235-237.

7. Gh. Mocanu, Sur quelques critères de commutativité dans les algèbres de Banach, Ann. Univ. București Mat.-Mec., 20 (1971), 127-129.

8. C. E. Rickart, General Theory of Banach Algebras, Princeton, Van Nostrand, 1960.

9. D. Z. Spicer, A commutativity theorem for Banach algebras, Colloquium Math., 27 (1973), 107-108.

10. E. Vesentini, On the subharmonicity of the spectral radius, Boll. Un. Mat. Ital., 4 (1968), 427-429.

11. E. Vesentini, Maximum theorems for spectra. Essays on topology and related topics dedicated to Georges de Rham, New-York, Springer-Verlag, 1970.

12. V. S. Vladimirov, Methods of the theory of functions of many complex variables, Cambridge, Mass. M.I.T. Press, 1966.

Received November 4, 1975.

Université Laval Québec, Canada 



\section{PACIFIC JOURNAL OF MATHEMATICS}

EDITORS

RICHARD ARENS (Managing Editor)

University of California

Los Angeles, California 90024

R. A. BEAUMONT

University of Washington

Seattle, Washington 98105
J. DugundJI

Department of Mathematics

University of Southern California

Los Angeles, California 90007

D. Gilbarg and J. Milgram

Stanford University

Stanford, California 94305

\section{ASSOCIATE EDITORS}

E. F. BECKENBACH

B. H. NeumanN

F. WOLF

K. YosHIDA

\section{SUPPORTING INSTITUTIONS}

UNIVERSITY OF BRITISH COLUMBIA

UNIVERSITY OF SOUTHERN CALIFORNIA

CALIFORNIA INSTITUTE OF TECHNOLOGY

UNIVERSITY OF CALIFORNIA

STANFORD UNIVERSITY

UNIVERSITY OF TOKYO

MONTANA STATE UNIVERSITY

UNIVERSITY OF UTAH

UNIVERSITY OF NEVADA

WASHINGTON STATE UNIVERSITY

NEW MEXICO STATE UNIVERSITY

UNIVERSITY OF WASHINGTON

OREGON STATE UNIVERSITY

UNIVERSITY OF OREGON

OSAKA UNIVERSITY

AMERICAN MATHEMATICAL SOCIETY
NAVAL WEAPONS CENTER

Printed in Japan by International Academic Printing Co., Ltd., Tokyo, Japan 


\section{Pacific Journal of Mathematics}

\section{Vol. 63, No. $1 \quad$ March, 1976}

Ralph Artino, Gevrey classes and hypoelliptic boundary value problems ....... 1

B. Aupetit, Caractérisation spectrale des algèbres de Banach commutatives .... 23

Leon Bernstein, Fundamental units and cycles in the period of real quadratic

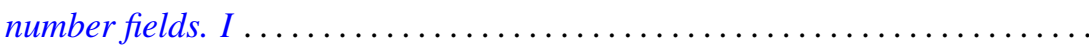

Leon Bernstein, Fundamental units and cycles in the period of real quadratic number fields. II.................................... 63

Robert F. Brown, Fixed points of automorphisms of compact Lie groups ........

Thomas Ashland Chapman, Concordances of noncompact Hilbert cube

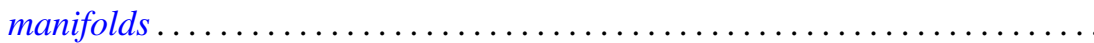

William C. Connett, V and Alan Schwartz, Weak type multipliers for Hankel

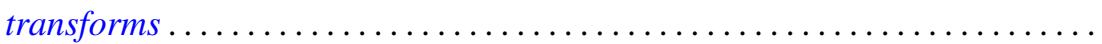

John Wayne Davenport, Multipliers on a Banach algebra with a bounded approximate identity .....................................

Gustave Adam Efroymson, Substitution in Nash functions ................ 137

John Sollion Hsia, Representations by spinor genera ..................

William George Kitto and Daniel Eliot Wulbert, Korovkin approximations in

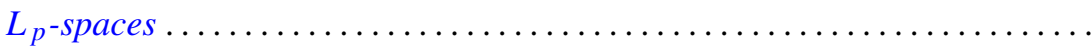

Eric P. Kronstadt, Interpolating sequences for functions satisfying a Lipschitz. condition ...........................................

Gary Douglas Jones and Samuel Murray Rankin, III, Oscillation properties of certain self-adjoint differential equations of the fourth order...

Takaŝi Kusano and Hiroshi Onose, Nonoscillation theorems for differential

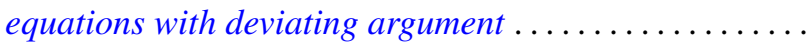

David C. Lantz, Preservation of local properties and chain conditions in commutative group rings. ...

Charles W. Neville, Banach spaces with a restricted Hahn-Banach extension property....

Norman Oler, Spaces of discrete subsets of a locally compact group ...

Robert Olin, Functional relationships between a subnormal operator and its minimal normal extension.

Thomas Thornton Read, Bounds and quantitative comparison theorems for nonoscillatory second order differential equations ...... .

Robert Horace Redfield, Archimedean and basic elements in completely distributive lattice-ordered groups...

Jeffery William Sanders, Weighted Sidon sets

Aaron R. Todd, Continuous linear images of pseudo-complete linear topological spaces.

J. Jerry Uhl, Jr., Norm attaining operators on $L^{1}[0,1]$ and the Radon-Nikodým property.

William Jennings Wickless, Abelian groups in which every endomorphism is a left multiplication. 